\title{
Omega-3 PUFAs and gut microbiota: A preventive approach for colorectal cancer
}

\author{
Marco Giammanco, ${ }^{1}$ Margherita Mazzola, ${ }^{2}$ Manfredi Marco Giammanco, ${ }^{3}$ Giovanni Tomasello, ${ }^{2}$ \\ Francesco Carini² \\ ${ }^{1}$ Department of Surgery, Oncologicical and Stomatological Sciences (Di.Chir.On.S), University Hospital Policlinico \\ Paolo Giaccone of Palermo, Palermo; ${ }^{2}$ Institute of Human Anatomy and Histology, Department of Biomedicine, \\ Neurosciences and Advanced Diagnostics, (BIND), University Hospital Policlinico Paolo Giaccone of Palermo, \\ Palermo; ${ }^{3}$ Department of Human Pathology in Adulthood and Childhood "G. Barresi", Medical School University of \\ Messina, Messina, Italy
}

\begin{abstract}
The influence of diet on the composition of the intestinal microbiota and related pathologies has been known for some time. Some classes of nutrients, such as fatty acids belonging to the omega 3 series, have particular effects on the bacteria that make up the intestinal microbiota. $\omega-3$ PUFAs affect the gut microbiota in three different ways: by modulating the type and abundance of intestinal bacteria, by regulate SCFAs levels, and by alter the levels of proinflammatory mediators. Through these modalities, $\omega-3$ PUFAs could be useful for the prevention of intestinal diseases such as Colorectal Cancer (CRC). The ability of $\omega-3$ PUFAs to modulate the intestinal inflammatory response, to preserve the integrity of the intestinal mucosa and to modulate the bacterial composition of the intestine, could be useful as a preventive strategic approach to hinder the development of CRC.
\end{abstract}

\footnotetext{
Correspondence: Margherita Mazzola, Institute of Human Anatomy and Histology, Department of Biomedicine, Neurosciences and Advanced Diagnostics, (BIND), University Hospital Policlinico Paolo Giaccone of Palermo, via del Vespro 137, 90127 Palermo, Italy.

E-mail: margheritamazzola@hotmail.it
}

Key words: $\omega-3$ PUFAs; colorectal cancer; prevention; gut microbiota; Mucosal Associated Lymphatic Tissue (MALT).

Conflicts of interest: The authors have no conflict of interest to declare.

Received for publication: 5 July 2021.

Revision received: 18 November 2021.

Accepted for publication: 20 November 2021.

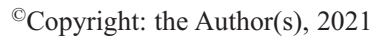

Licensee PAGEPress, Italy

Journal of Biological Research 2021; $94: 9954$

doi:10.4081/jbr.2021.9954

This article is distributed under the terms of the Creative Commons Attribution Noncommercial License (by-nc 4.0) which permits any noncommercial use, distribution, and reproduction in any medium, provided the original author(s) and source are credited.

\section{Introduction}

The gut microbiota consists of a population of various and highly dynamic microorganisms, whose composition is characterized by the presence of 500 to 1,000 different bacterial species, and a low number virus and fungal species. ${ }^{1,2}$ Our intestine hosts trillions of microorganisms, the highest concentration $\left(9 \times 10^{13-14}\right)$ being found in the colon, with a very high bacterial diversity. 1,3 The main bacteria present in the colon belong to four main phyla: Firmicutes (64\%), Bacteroidetes (23\%), Actinobacteria and Proteobacteria. ${ }^{4}$ The intestinal microbiota is formed at the time of birth, during the passage through the birth canal. At this time, the neonatal intestine is colonized by the maternal flora, composed of Bacteroidetes, Bifidobacterium, Prevotella and Lactobacillus spp. ${ }^{3,5}$ The intestinal microbiota regulates several important biological processes, including fermentation of amino acids and saccharides with the subsequent production of Short-Chain Fatty Acids (SCFA), succinate, ethanol, amines, lactate, phenols, thiols and indoles, of hydrogen gas (such as H2S acetate and methane), degradation of undigested proteins and carbohydrates, and the transformation of bile acids. ${ }^{3,5}$ Furthermore, gut microbiota plays a fundamental role for the health of our organism. It regulates the proliferation of pathogenic bacteria present in the intestinal tract (such as Clostridia or Colibacillacea), stimulates the immune system, regulates the absorption of nutrients, regulates host metabolism, promote the production of vitamins such as vitamin $\mathrm{K}$ and biotin, and enzymes and the synthesis of compounds useful for the trophism of the colon mucosa and that are essential for cell renewal. ${ }^{3,5,6}$ On the other hand, any alteration in intestinal eubiosis can lead to an altered composition of gut microbiota, known as dysbiosis, a condition that determines changes of the tight intercellular junctions responsible for maintaining the integrity of the intestinal mucosa and its permeability which is fundamental to prevent the access of pathogens. ${ }^{6}$ In case of severe dysbiosis, Mucosal Associated Lymphatic Tissue (MALT) once activated triggers the inflammatory cascade (leukocytes, cytokines, TNF- $\alpha$ ) and consequently a massive tissue injury and a possible fostering of colorectal carcinogenesis process. ${ }^{7}$ Dysbiosis develops due to a variety of factors including physical and psychological stress, environmental stress, diet, and drug intake. ${ }^{3}$ In particular, diet appears to be one of the determining factors for the composition of the intestinal microbiota. ${ }^{3,8} \mathrm{~A}$ diet rich in saturated fats and simple sugars and a poor content of fruits and vegetables is known to increase the risk of metabolic disorders. ${ }^{8}$ Furthermore, it is well established that 
diet has a great influence (57\% vs $12 \%$ for genetic factors) on the composition of the microbiota. Many studies have shown that long-term consumption of large amounts of fat and sugar results in a worsening of dysbiosis and in a changed composition of the microbiota, as well as in the production of endotoxins. ${ }^{9}$ Moreover, this type eating-habit leads to changes in the intestinal mucosa, which becomes thinner and thinner, more permeable to pathogens and antigens and more susceptible to the establishment of lowgrade but persistent inflammation..$^{3,9}$

The association between diet-induced dysbiosis and Colorectal Cancer (CRC) was highlighted by a study of the intestinal microbial structures and faecal metabolite profiles among populations consuming different types of diet. ${ }^{10}$ For instance, the intestinal microbiota of rural Africans, whose diet is rich in fibre and low in fat, is characterized by a predominance of the genus Prevotella, involved in the degradation of starches, hemicelluloses and xylans. ${ }^{10}$ On the other hand, the microbiota of the American population is predominated by the Bacteroides genus with an increased abundance of potentially pathogenic proteobacteria, such as Escherichia Coli and Acinetobacter. ${ }^{10}$ These microbial and structural differences parallel the low incidence of colorectal cancer in Africa compared to Western countries. ${ }^{10}$ There are also differences in faecal metabolites profile, with higher SCF in Native Africans and higher secondary bile acids in African Americans. ${ }^{10}$ These observational results were consistent with those from a study showing that the switching of African-Americans diet to a high-fibre, low-fat diet for 2 weeks, increased SCFA production, suppresses secondary bile acid synthesis, and reduces inflammation of the colon mucous membranes and proliferation of tumour markers. In contrast to the tumour, promoting effects of secondary bile acids, SCFs have been shown to protect against colorectal cancer through their beneficial effects on immune and metabolic pathways. ${ }^{10}$

Other studies have suggested that a compromission of the colonic mucus layer, a physical barrier that separates trillions of gut bacteria from the host, may represent a potential mechanism by which a direct interaction between Western diet and gut microbiota can foster carcinogenesis. ${ }^{11,12}$ In fact, the excess of saturated fats and refined sugars slows the growth rate of mucus and increases the penetration of the intestinal barrier by bacteria. ${ }^{11,12}$ It also causes a gradual decrease in SCFA-producing bacteria, such as Bifidobacterium and Bacteroidales, and an increase in Firmicutes. ${ }^{11,12}$

Given the important role of the diet on intestinal dysbiosis and the genesis of $\mathrm{CRC}$, recent studies have investigated the role of particular polyunsaturated fatty acids present in the diet on the intestinal microbiota and on colorectal carcinogenesis.

In this review we will talk about one of the fundamental components of the Mediterranean diet, the fatty acids of the omega 3 series, their role in carcinogenesis and their interaction with the intestinal microbiota.

\section{(-3 PUFA: characteristics and role in pathological conditions}

Long-chain Polyunsaturated Fatty Acids (PUFAs) are organic substances characterized by a high number of carbon atoms, some of which are linked with a double bond (hence the definition of unsaturated fatty acids). ${ }^{13}$ From a nutritional point of view, the most important PUFAs are represented by omega $3(\omega-3)$ series, which include $\alpha$-linolenic (ALA; 18:3), eicosapentaenoic acid (EPA; 20:5) and docosahexaenoic acid (DHA; 22:6). Fishery products from fatty fish such as salmon, sardines, mackerel and blue fish in general, are, by far, the most important source of $\omega-3$ PUFA, as absolute quantity and in percentage. ${ }^{13}$ EPA and DHA come mainly from the consumption of fish that, thanks to a diet based on microalgae rich in DHA and EPA and phytoplankton, accumulates $\omega-3$ PUFA. ${ }^{1}$ ALA is mainly obtained through the intake of flax seeds, chia seeds and walnuts. EPA and DHA can be synthesized in the human body by using ALA as a precursor. However, this conversion account only for $6 \%$ of EPA and $<4 \%$ of DHA. ${ }^{1}$ For this reason, $\omega-3$ PUFAs are considered essential fatty acids to be taken through the diet. ${ }^{1}$ The interest in the $\omega$-3 PUFA fatty acids originated from the epidemiological observations of the populations of the west coast of Greenland, ${ }^{14,15}$ whose diet is characterized by a high fat content and a reduced consumption of fruit and vegetables. Despite this type of diet, which is markedly different from the Mediterranean diet, these populations have a reduced incidence of dyslipidemia and mortality from myocardial infarction compared to other European populations. ${ }^{13}$ The explanation for this phenomenon lies in the fact that the high consumption of fish, and therefore $\omega-3$ PUFA, results in a higher content of EPA and a lower content of Arachidonic Acid (AA) in the membrane phospholipids of platelets. ${ }^{13}$ Since TXA3, a non-aggregatory thromboxan, is formed from EPA, the increase in the EPA/AA platelet ratio leads to a lower efficiency of platelet aggregation, a lower tendency to form thrombi and, consequently, a lower incidence of atherosclerosis. ${ }^{13}$ Therefore, the administration of $\omega-3$ PUFA may reduce the progression of atherosclerotic lesions and improve the haemodynamic and physical properties of the large arteries. ${ }^{13}$ According to the Italian Society of Human Nutrition (SINU), the levels of reference intake for the Italian population (LARN) for $\omega-3$ PUFAs $^{16}$ are those shown in Table 1.

Studies carried out on the populations of Greenland, ${ }^{14,15}$ have shown, in addition to the usefulness of $\omega-3$ PUFAs in reducing the risk of coronary heart disease, ${ }^{17}$ these molecules are also effective in improving parameters associated with the Metabolic Syndrome (MetS). ${ }^{18}$ MetS is a condition characterized by the combination of three or more different components of cardiometabolic risk factors, including obesity, hypertension, dyslipidemia and impaired glucose tolerance. ${ }^{18}$ Patients with MetS are at high risk of cardiovascular disease and type 2 diabetes (DM-2). ${ }^{19}$ As the mechanisms responsible for MetS involves both genetic and acquired factors, an effective therapeutic strategy in MetS patients may be based on lifestyle modifications, weight loss and a regular physical activity associated with the administration of supplements, in particular PUFA $\omega-3 .{ }^{19,20}$ Consistent with these observations, data from some Randomized Control Trial (RCT) have shown that a 12 weeks supplementation with $\omega$-3 PUFA (1.2 g/day) resulted in a significant reduction of circulating concentrations of triglycerides and nonesterified fatty acids while, the prevalence of MetS decreased by $10.5 \% .^{21,22}$ Another RCT in which patients with MetS were given $3 \mathrm{~g}$ of fish oil up to 90 days, reported, a significant reduction in diastolic blood pressure compared to the baseline value in these patients. ${ }^{19}$ Other studies have also provided evidence on the use-

Table 1. LARN for $\omega$-3 PUFAs.

\begin{tabular}{lc} 
Age & \multicolumn{1}{c}{ mg/day } \\
Infants & EPA-DHA 250 mg+ DHA $100 \mathrm{mg}$ \\
Children - teenagers & EPA-DHA 250 mgl-2 anni +DHA 100 mg \\
\hline Adults - elderly & EPA-DHA 250 mg \\
Pregnancy - breastfeeding & EPA-DHA 250 mg+DHA 100-200 mg \\
\hline
\end{tabular}


fulness of $\omega$-3 PUFAs in the treatment of mood disorders, as their intake is correlated with a decrease in anxiety symptoms. ${ }^{23}$ These findings have suggested the clinical use $\omega-3$ PUFAs also in the treatment of major depressive disorder. ${ }^{24}$

Interestingly, recent experimental studies have suggested a possible anticancer activity of $\omega-3$ PUFAs. ${ }^{25}$ In particular, these studies have provided evidence on the capability of $\omega-3$ PUFAs to inhibit some of the multiple oncogenic pathways which lead to the inhibition of proliferation and activation of apoptosis. ${ }^{25,26}$ In this setting, recent in vitro studies have highlighted the fact that, $\omega-3$ PUFAs may thwart the growth of hormone resistant tumours. ${ }^{1,26}$ Furthermore, these studies also showed a preferential inhibition of $\omega-3$ PUFAs for triple negative breast cancer cells rather than luminal breast cancer cells. ${ }^{1,27}$ Modulation of molecular pathways promoting inflammation is another supposed mechanism by which $\omega$ 3 PUFAs can exert anticancer effects. ${ }^{28}$ Consistent with these observations, some, in vivo studies have reported that Specialized Pro-resolving lipid Mediators (SPMs), which include EPA and DHA-derived resolvins, protectins and maresins, may inhibit chemically-induced colitis in rodents. These SPMs act by suppressing NF-kB nuclear translocation, thus reducing the expression of TNF- $\alpha$ and other pro-inflammatory cytokines while, they increase the production of anti-inflammatory cytokines, such as interleukin-10, in monocytes. ${ }^{29-32}$

SPMs also regulate intestinal bacterial populations by limiting collateral tissue damage that often occurs during the elimination of bacterial pathogens. They also stimulate the production of antimicrobial peptides in the intestinal mucosa, can improve phagocytosis of bacteria by inhibiting the production of interleukin-12, and suppress the migration of dendritic cells..$^{29,30,33}$

\section{(-3 PUFA: influence on the intestinal microbiota}

Like all dietary components, $\omega-3$ PUFAs may also influence the composition of the intestinal microbiota. It has previously been observed that -3 PUFAs play a role in the regulation of intestinal bacterial populations and in the promotion of intestinal immune homeostasis, through their endogenous metabolites. ${ }^{33}$

$\omega-3$ PUFAs affect the gut microbiota in three different ways; first, by modulating the type and abundance of intestinal bacteria. For example, compared to saturated fatty acids, $\omega-3$ PUFAs influence positively the intestinal microbiota by increasing the presence of Lactobacillus and Bifidobacteria. ${ }^{34-37}$ This phenomenon has been reported to be associated with a low-grade inflammation, and a lower presence of Helicobacter and Fusobacteria nucleatum. ${ }^{34-37}$

Furthermore, $\omega-3$ PUFAs could exert beneficial effects on the intestinal microbiota by decreasing the growth of Enterobacteria, by increasing the growth rate of Bifidobacteria, and subsequently, by inhibiting the inflammatory response associated with metabolic endotoxemia. ${ }^{38} \omega-3$ PUFAs derived from the diet, are partially metabolized in the distal intestine, by anaerobic bacteria such as Bifidobacteria and Lactobacilli. ${ }^{39}$ Experimental in vivo studies in animal models have shown that these metabolic processes determine marked changes in the microbial composition of the intestine. ${ }^{39}$ The increase in the population of beneficial bacteria results in an improvement of the intestinal microenvironment and the barrier function of the intestinal mucosa, an increase of the thickness of the intestinal mucosa, and the fostering of weight loss by modulating the expression of genes related to fat metabolism. ${ }^{40}$ The effects of $\omega-3$ PUFAs on the intestinal microbial population also result in the prevention and/or improvement of specific symp- toms of various diseases related to intestinal dysbiosis. Firmicutes and Bacteroidetes are two major dominant bacterial phyla of the human gut microbiota. ${ }^{41}$

The Firmicutes-to-Bacteroidetes ratio (F/B ratio) has been reported to be associated with obesity. ${ }^{42}$ In fact, while in healthy subjects this ratio ranges from $1: 1$ to $3: 1$, in $35 \%$ of obese or overweight subjects there is an unbalanced ratio to favour of the Firmicuti, from 3: 1 up to 25: $1 .^{43}$ On the other hand, Onishi et al. ${ }^{44}$ have shown that $\omega-3$ PUFAs are able to hinder the decrease in the $\mathrm{F} / \mathrm{B}$ ratio observed in mice fed a high-fat diet. A second way in which $\omega-3$ PUFAs may affect the intestinal microbiota is related to their capability to alter the levels of proinflammatory mediators, such as endotoxins, resulting from bacterial Lipopolysaccharide (LPS) and Interleukin-17 (IL-17). ${ }^{45,46}$ Following the occurrence of dysbiosis, LPS can cross the intestinal wall, due to the loss of the integrity of the intestinal barrier, thus causing further damages. The increased intestinal permeability, in turn, results in an accumulation of toxic bacterial products such as LPS and bacterial DNA in the systemic circulation. This phenomenon can potentially elicit an inflammatory response. ${ }^{45,46}$ Consumption of $\omega-3$ PUFAs inhibits LPS-induced pro-inflammatory cytokine production in human blood monocytes, relieving intestinal inflammation, by inhibiting LPS induced activation of NF- $\mathrm{KB}$ signalling pathways. ${ }^{47}$ These observations are in line with the findings from other in vitro studies showing that macrophage incubation with $\omega$-3 PUFAs reduces LPS-induced MAPK activity and decreases the expression of proinflammatory mediators, such as TNF- $\alpha .{ }^{48} \omega-3$ PUFAs also promote the release of large amounts of anti-inflammatory cytokines from resident macrophages, such as Interleukin-10 (IL10), the induction of regulatory $\mathrm{T}$ lymphocytes (Treg) and prevent an excessive development of T-helper 17 cells (Th17) and that of IL-17, a pro-inflammatory cytokine produced mainly by Th17 cells, which causes tissue inflammation. Therefore, $\omega-3$ PUFAs can reduce intestinal inflammation by increasing the differentiation of Tregs and by decreasing the production of IL-17.49 A third way in which $\omega-3$ PUFAs may affects gut microbiota relies on the ability of these molecules to regulate SCFAs levels. ${ }^{37}$ The production of SCFAs, including butyrate, propionate and acetate, plays an essential role in maintaining a healthy mucosa and in the production of anti-inflammatory interleukins. SCFAs derives from the bacterial fermentation of carbohydrates from food. This process occurs in the colon, under anaerobic conditions, with the contribute of Lactobacilli and Bifidobacteria. Among the SCFAs, butyric acid is the preferred energy source for colon cells, where it controls the state of inflammation, proliferation, differentiation and apoptosis. It also has a role in strengthening the defensive barrier of the colon by increasing the production of mucin and antimicrobial peptides and, by decreasing the permeability of the intestinal epithelial and by increasing the expression of the proteins constituting the tight junctions. ${ }^{3}$ Numerous studies have shown that increasing the daily intake of $4 \mathrm{~g}$ of mixed $\omega-3$ PUFAs (DHA and EPA) significantly increased the density of butyrate-producing bacteria. ${ }^{37}$ The mechanisms mentioned above could be useful in the prevention and adjuvant treatment of CRC.

\section{$\omega-3$ PUFAs: role in the prevention of colorectal cancer}

The ability of $\omega$-3 PUFAs to modulate the intestinal inflammatory response, to preserve the integrity of the intestinal mucosa and to modulate the bacterial composition of the intestine, could be 
useful as a preventive strategic approach to hinder the development of CRC. This malignant disease represents one of the most common cancers in the world population, with a multifactorial pathogenesis linked, among other things, to environmental factors, a sedentary lifestyle and an incorrect diet. ${ }^{50}$ The inverse correlation between $\omega$-3 PUFA intake and CRC development has been highlighted by several human studies that have analyzed the relationship between microbiota and $\omega-3$ PUFA in some stages of carcinogenesis. ${ }^{51}$ In this setting, Prossomariti et al., ${ }^{52}$ showed that in patients with ulcerative colitis (RCU, a disease which is serious risk factor for CRC), supplementation with EPA ( $2 \mathrm{~g} /$ day for 90 days) led to an improvement in endoscopic inflammation and to the modulation of the intestinal microbiota. In particular, the genus Parabacteroides, which has been found to be lower in patients with RCU, was significantly increased after EPA supplementation. Conversely, Clostridium spp. and Bacteroides, which are known to trigger mucolytic metabolism, were found to be decreased after supplementing with EPA. ${ }^{52}$ Other numerous epidemiological and preclinical studies further support the preventive effects of $\omega-3$ PUFA on CRC. For example, in the VITamins And Lifestyle (VITAL) cohort study, subjects who routinely took fish oil supplements had lower risks of developing CRC than those who didn't take supplements. ${ }^{53,54}$ Another study, carried out by the European Prospective Investigation into Cancer and Nutrition (EPIC) showed that increased consumption of $\omega-3$ PUFA reduces the risks of CRC. ${ }^{55}$ The results of a randomized, double-blind, placebo-controlled study, demonstrated that EPA intake was associated with a reduced number and size of polyps in patients with Familial Adenomatous Polyposis (FAP) ${ }^{56}$ In particular, this study showed that adding EPA at a dose of $2 \mathrm{~g}$ /day for 6 months reduced the number and size of polyps by $20-30 \%$. These results, are comparable to that obtained by other studies following long-term treatments with cyclooxygenase-2 inhibitors. ${ }^{11}$ Besides CRC prevention, $\omega-3$ PUFAs have been reported to be useful as adjuvants to chemotherapy in the treatment of advanced disease. ${ }^{57}$ In this context, clinical observations have reported that the increased $\omega$-3 PUFA intake was also associated with improved disease-free survival in patients with stage III CRC. ${ }^{57}$

Moreover, the results from a double-blind, randomized, placebo-controlled phase II study, carried out by Cockbain et al. ${ }^{58}$ demonstrated that the oral administration of EPA to patients with advanced CRC who underwent liver resection due to liver metastases was associated with increased overall survival. Furthermore, some clinical investigations have also shown a significant correlation between $\omega-3$ PUFA intake and survival of CRC patients. ${ }^{34}$ Finally, a study carried out on two groups of women that were treated with $<0.1 \mathrm{~g} /$ day or with $0.3 \mathrm{~g} /$ day of $\omega-3$ PUFA respectively, showed a decreased rate of mortality in subjects administered with the highest dose. This reduction was more marked when $\omega-3$ PUFA consumption was further increased to $0.15 \mathrm{~g} / \mathrm{d} .^{34}$

In recent years we have also seen how nutrition, including the administration of $\omega-3$ PUFA, is able to influence the health conditions of individuals and the susceptibility to diseases by defining the metabolic response and gene expression. In fact, epigenetic modifications can play a significant role in the onset and pathogenesis of CRC, with mechanisms such as DNA methylation and chromatin remodeling. $\omega$-3 PUFA, however, act differently as their epigenetic action does not involve DNA methylation. In fact, it has been seen that the intake of PUFA in association with dietary fibres upregulates the nuclear receptors dependent on the EPA and DHA ligand and supports the increase in butyrate production through the fermentation of fibres, which directly and indirectly modifies the acetylation of histones. These combinatorial effects improve the mitochondrial shuttle of L-carnitine, inhibit lipogenesis and promote the accumulation of acetyl CoA-dependent beta-oxidation. The increased beta-oxidation of the mucosa maintains a hypoxic environment in the intestine and promotes the growth of obligate anaerobic bacteria and inhibits dysbiotic microbial expansion. ${ }^{59}$ This paves the way for future studies on $\omega-3$ PUFA, CRC and epigenetic modifications.

\section{Conclusions}

Daily consumption and the possible integration of $\omega-3$ PUFA appears to exerts beneficial effects on human. Primary and secondary prevention against cardiovascular and ischemic diseases can be made by maintaining adequate circulating levels of $\omega-3$. This adaptation is also effective to improve the parameters related to cholesterol and triglycerides. Adequate consumption of $\omega-3$ PUFA may also improves the blood and pressure parameters of subjects affected by MetS, while it is still not clear whether the additional supplementation with $\omega-3$ PUFA alone can have preventive effects on the development of MetS. The studies that correlate the consumption of $\omega-3$ PUFA to the inhibition of carcinogenesis, and in particular to $\mathrm{CRC}$, are interesting. This multifactorial pathology recognizes, among its causes, an alteration of the intestinal microbiota, referred as dysbiosis. $\omega-3$ PUFAs influence the intestinal microbiota through three different mechanisms: i) by modulating the type and quantity of bacteria present in the intestine; ii) by reducing the inflammatory phenomena caused by intestinal dysbiosis; iii) by regulating the levels of SCFAs in the intestine. These effects have been also shown to be effective in the prevention and/or treatment and survival of CRC. In fact, it has been observed that the incidence of CRC is lower in patients who habitually consume $\omega-3$ PUFA. However, these results do not prove a direct relationship between $\omega-3$ PUFA and prevention of CRC as several other factors may contribute to the etiopathogenesis of CRC. It was also possible to observe how patients with CRC or FAP had beneficial effects in terms of reduction of neoplastic and paraneoplastic lesions and in terms of survival. We can therefore conclude that the dietary intake and the possible daily supplementation of $\omega$-3 PUFA have beneficial effects on human health.

\section{References}

1. Weir TL, Trikha SRJ, Thompson HJ. Diet and cancer risk reduction: The role of diet-microbiota interactions and microbial metabolites. Semin Cancer Biol 2021;70:53-60.

2. Gentile CL, Weir TL. The gut microbiota at the intersection of diet and human health. Science 2018;362:776-80.

3. Tomasello G, Mazzola M, Leone A, et al. Nutrition, oxidative stress and intestinal dysbiosis: Influence of diet on gut microbiota in inflammatory bowel diseases. Biomed Pap Med Fac Univ Palacky Olomouc Czech Repub 2016;160:461-6.

4. Sartor RB, Mazmanian SK. Intestinal microbes in inflammatory bowel diseases. Am J Gastroenterol Suppl 2012;1:15-21.

5. Tralongo P, Tomasello G, Sinagra E, et al. The role of butyric acid as a protective agent against inflammatory bowel diseases. Euromediter Biomed J 2014;9:24-35.

6. Tomasello G, Tralongo P, Damiani P, et al. Dismicrobism in inflammatory bowel disease and colorectal cancer: Changes in response of colocytes. World J Gastroenterol 2014;20:18121-30. 
7. Tomasello G, Zeenny MN, Giammanco M, et al. Intestinal microbiota mutualism and gastrointestinal diseases. Euromediterr Biomed J 2015;10;65-75.

8. Mazzola M, Carini F, Damiani P, et al. Inflammatory bowel disease and colorectal cancer, nutraceutical aspects. Euromediterr Biomed J 2016;11:123-9.

9. Agus A, Denizot J, Thévenot J, et al. Western diet induces a shift in microbiota composition enhancing susceptibility to adherent-invasive E. coli infection and intestinal inflammation. Sci Rep 2016;6:19032.

10. O'Keefe SJ, Li JV, Lahti L, et al. Fat, fibre and cancer risk in African Americans and rural Africans. Nat Commun 2015;6:6342.

11. Song M, Chan AT. Environmental factors, gut microbiota, and colorectal cancer prevention. Clin Gastroenterol Hepatol 2019;17:275-89.

12. World Cancer Research Fund/American Institute for Cancer Research. Continuous update project report: diet, nutrition, physical activity and colorectal cancer. Available from: wcrf.org/colorectal-cancer-2017

13. La Guardia M, Giammanco S, Di Majo D, et al. Omega 3 fatty acids: biological activity and effects on human health. Panminerva Med 2005;47:245-57.

14. Bang HO, Dyerberg J, Nielsen AB. Plasma lipid and lipoprotein pattern in Greenlandic West-coast Eskimos. Lancet 1971;1:1143-5

15. Dyerberg J, Bang HO. Haemostatic function and platelet polyunsaturated fatty acids in Eskimos. Lancet 1979;2:433-5.

16. Società Italiana di Nutrizione Umana-SINU, 2014. LARN Livelli di assunzione di riferimento per la popolazione italiana: LIPIDI. Available from: https://sinu.it/2019/07/09/lipidi/

17. Sudheendran S, Chang C and Deckelbaum, R. N-3 vs. saturated fatty acids: effects on the arterial wall. Prostaglandins Leukotrienes Essential Fatty Acids 2010;82:205-9.

18. Expert Panel on Detection, Evaluation, and Treatment of High Blood Cholesterol in Adults. Executive summary of the third report of the national cholesterol education program (NCEP) expert panel on detection, evaluation, and treatment of high blood cholesterol in adults (adult treatment panel III). JAMA 2001;285:2486-97.

19. Guo XF, Li X, Shi M, Li D. n-3 polyunsaturated fatty acids and metabolic syndrome risk: A meta-analysis. Nutrients 2017;9:703.

20. Grundy SM. Diagnosis and management of the metabolic syndrome an American heart association/national heart, lung, and blood institute scientific statement. Curr Opin Cardiol 2005;21:1-6.

21. Dembinska A. Effects of dietary fat modification on insulin sensitivity and on other risk factors of the metabolic syndrome-LIPGENE: A European randomized dietary intervention. Int J Obes 2011;35:800-9.

22. Paniagua JA, Pérez-Martinez P, Gjelstad IM, et al. A low-fat high-carbohydrate diet supplemented with long-chain n-3 PUFA reduces the risk of the metabolic syndrome. Atherosclerosis 2011;218:443-50.

23. Su KP, Tseng PT, Lin PY, et al. Association of use of omega-3 polyunsaturated fatty acids with changes in severity of anxiety symptoms. JAMA Network Open 2018;1:e182327

24. Hallahan B, Ryan T, Hibbeln JR, et al. Efficacy of omega-3 highly unsaturated fatty acids in the treatment of depression. Br J Psychiat 2016;209:192-201.

25. Zhu Z, Jiang W, McGinley JN, et al. Mammary gland density predicts the cancer inhibitory activity of the N-3 to N-6 ratio of dietary fat. Cancer Prev Res (Phila) 2011;4:1675-85.
26. D'Eliseo D, Velotti F. Omega-3 fatty acids and cancer cell cytotoxicity: Implications for multi-targeted cancer therapy. J Clin Med 2016;5:15.

27. Pogash TJ, El-Bayoumy K, Amin S, et al. Oxidized derivative of docosahexaenoic acid preferentially inhibit cell proliferation in triple negative over luminal breast cancer cells. In Vitro Cell Dev Biol Anim 2015;51:121-7.

28. Khadge S, Sharp JG, Thiele GM, et al. Fatty acid mediators in the tumor microenvironment. Adv Exp Med Biol 2020;1259:125-53

29. Ishida T, Yoshida M, Arita M, et al. Resolvin E1, an endogenous lipid mediator derived from eicosapentaenoic acid, prevents dextran sulfate sodium-induced colitis. Inflamm Bowel Dis 2010; $16: 87-95$.

30. Marcon R, Bento AF, Dutra RC, et al. Maresin 1, a proresolving lipid mediator derived from omega-3 polyunsaturated fatty acids, exerts protective actions in murine models of colitis. J Immunol 2013;191:4288-98.

31. Gu Z, Lamont GJ, Lamont RJ, et al. Resolvin D1, resolvin D2 and maresin 1 activate the GSK3 $\beta$ anti-inflammatory axis in TLR4-engaged human monocytes. Innate Immun 2016;22:186-95.

32. Chiang N, De la Rosa X, Libreros S, Serhan CN. Novel Resolvin D2 receptor axis in infectious inflammation. J Immunol 2017;198:842-51.

33. Gibson GR, Hutkins R, Sanders ME, et al. Expert consensus document: The International Scientific Association for Probiotics and Prebiotics (ISAPP) consensus statement on the definition and scope of prebiotics. Nat Rev Gastroenterol Hepatol 2017;14:491-502.

34. Chapkin RS, Navarro SL, Hullar MAJ, Lampe JW. Diet and gut microbes act coordinately to enhance programmed cell death and reduce colorectal cancer risk. Dig Dis Sci 2020;65:840-51.

35. Patterson E, O'Doherty RM, Murphy EF, et al. Impact of dietary fatty acids on metabolic activity and host intestinal microbiota composition in C57BL/6J mice Br J Nutr 2014;111:1905-17.

36. Tabbaa M, Golubic M, Roizen MF, Bernstein AM. Docosahexaenoic acid, inflammation, and bacterial dysbiosis in relation to periodontal disease, inflammatory bowel disease, and the metabolic syndrome. Nutrients 2013;5:3299-310.

37. Fu Y, Wang Y, Gao H, et al. Associations among dietary Omega-3 polyunsaturated fatty acids, the gut microbiota, and intestinal immunity. Mediators Inflamm 2021;2021:8879227.

38. Cao W, Wang C, Chin Y, et al. DHA-phospholipids (DHA-PL) and EPA-phospholipids (EPA-PL) prevent intestinal dysfunction induced by chronic stress. Food Function 2019;10:277-88.

39. Lauridsen C. Effects of dietary fatty acids on gut health and function of pigs pre- and post-weaning. J Animal Sci 2020;98:skaa086.

40. Warner DR, Warner JB, Hardesty JE, et al. Decreased $\omega-6: \omega-3$ PUFA ratio attenuates ethanol-induced alterations in intestinal homeostasis, microbiota, and liver injury. J Lipid Res 2019;60:2034-49.

41. Rinninella E, Raoul P, Cintoni M, et al. What is the healthy gut microbiota composition? A changing ecosystem across age, environment, diet, and diseases. Microorganisms 2019;7:14.

42. Crovesy L, Masterson D, Rosado EL. Profile of the gut microbiota of adults with obesity: a systematic review. Eur J Clin Nutr 2020;74:1251-62.

43. PM Keller, M. Hombach, GV Bloemberg. 16S-rRNA-Genbasierte Identifikation bakterieller infektionen. BIOspektrum 2010:755-8. 
44. Onishi JC, Campbell S, Moreau M, et al. Bacterial communities in the small intestine respond differently to those in the caecum and colon in mice fed low- and high-fat diets. Microbiology (Reading) 2017;163:1189-97.

45. Schoeler M, Caesar R. Dietary lipids, gut microbiota and lipid metabolism. Rev Endocrine Metabolic Disord 2019;20:461-72.

46. Hutchinson AN, Tingö L, Brummer RJ. The potential effects of probiotics and $\omega-3$ fatty acids on chronic low-grade inflammation. Nutrients 2020;12:2402.

47. Husson MO, Ley D, Portal C, et al. Modulation of host defence against bacterial and viral infections by omega-3 polyunsaturated fatty acids. J Infect 2016;73:523-35.

48. Babcock TA, Kurland A, Helton WS, et al. Inhibition of activator protein-1 transcription factor activation by omega-3 fatty acid modulation of mitogen-activated protein kinase signaling kinases. JPEN J Parenter Enteral Nutr 2003;27:176-80.

49. Kim JY, Lim K, Kim KH, et al. N-3 polyunsaturated fatty acids restore Th17 and Treg balance in collagen antibody-induced arthritis. PLoS One 2018;13:e0194331.

50. Carini F, Mazzola M, Rappa F, et al. Colorectal Carcinogenesis: Role of Oxidative Stress and Antioxidants. Anticancer Res 2017;37:4759-66.

51. Costantini L, Merendino N. Polyunsaturated fatty acids and microbiota relationship: Implications in cancer onset and treatment. J Clin Med 2020;9:3490.

52. Prossomariti A, Scaioli E, Piazzi G, et al. Short-term treatment with eicosapentaenoic acid improves inflammation and aspects colonic differentiation markers and microbiota in patients with ulcerative colitis. Sci Rep 2017;7:7458.

53. Tu M, Wang W, Zhang G, Hammock BD. $\omega-3$ Polyunsaturated fatty acids on colonic inflammation and colon cancer: Roles of lipid-metabolizing enzymes involved. Nutrients 2020;12:3301.

54. Kantor ED, Lampe JW, Peters U, et al. Long-chain omega-3 polyunsaturated fatty acid intake and risk of colorectal cancer. Nutr Cancer 2014;66:716-27.

55. Aglago EK, Huybrechts I, Murphy N, et al. Consumption of fish and long-chain n-3 polyunsaturated fatty acids is associated with reduced risk of colorectal cancer in a large european cohort. Clin Gastroenterol Hepatol 2020;18:654-66.

56. West NJ, Clark SK, Phillips RK, et al. Eicosapentaenoic acid reduces rectal polyp number and size in familial adenomatous polyposis. Gut 2010;59:918-25

57. Michalak A, Mosińska P, Fichna J. Polyunsaturated fatty acids and their derivatives: Therapeutic value for inflammatory, functional gastrointestinal disorders, and colorectal cancer. Front Pharmacol 2016;7:459.

58. Cockbain AJ, Volpato M, Race AD, et al. Anticolorectal cancer activity of the omega-3 polyunsaturated fatty acid eicosapentaenoic acid. Gut 2014;63:1760-8.

59. Chapkin RS, Navarro SL, Hullar MAJ, Lampe JW. Diet and Gut Microbes Act Coordinately to Enhance Programmed Cell Death and Reduce Colorectal Cancer Risk. Dig Dis Sci 2020;65:840-51. 